\title{
EFEITO RESIDUAL EM CAMPO DO IMIDACLOPRIDO NO CONTROLE DO PULGÃO-DO-PINUS (Cinara spp.), SOB DUAS MODALIDADES DE APLICAÇÃO NO VIVEIRO DE MUDAS
}

\author{
RESIDUAL EFFECT IN FIELD OF IMIDACHLORPRID IN THE CONTROL OF \\ THE PINE APHID (Cinara SPp.), UNDER TWO MODALITIES OF APPLICATION \\ IN THE NURSERY OF SEEDLINGS
}

\author{
Álvaro Boson de Castro FARIA ${ }^{1}$ \\ Nilton José SOUSA ${ }^{2}$
}

\begin{abstract}
RESUMO
A presença de pulgões do gênero Cinara (Curtis, 1835) em plantios de Pinus foi detectada no Brasil em 1996 pela equipe da Embrapa-CNPF, e os ataques podem favorecer a mortalidade de mudas no plantio. Neste sentido, técnicas e estratégias de controle desta praga devem considerar os parâmetros ambientais, econômicos e sociais dos plantios florestais, em virtude do contexto do "Manejo Integrado de Pragas". Com base nestas premissas, os objetivos deste trabalho foram: determinar a viabilidade de uso da molécula imidacloprido na proteção de mudas de Pinus taeda L. contra a presença do pulgão-do-pinus (Cinara spp.), sob duas modalidades de aplicação no viveiro de mudas. A metodologia consistiu em um delineamento experimental inteiramente casualisado com três tratamentos (imidacloprido aplicado via rega; imidacloprido associado ao gel condicionador de solo e testemunha) e seis repetições. A infestação foi avaliada através dos parâmetros prevalência e incidência. O efeito residual do imidacloprido aplicado sobre mudas em viveiro, quer na forma de rega ou associado ao gel condicionador de solo, se prolonga por até 80 dias após o plantio das mudas em campo. Este efeito não impede a infestação inicial das mudas pelo pulgão-do-pinus (Cinara spp.), porém é capaz de evitar a expansão populacional das colônias.
\end{abstract}

Palavras-chave: Inseticida sistêmico; imidacloprido; manejo integrado de pragas florestais.

\begin{abstract}
The pine aphid (Cinara Curtis, 1835) was detected in Brazil in 1996 by researchers of Brazilian Agricultural Research Corporation. Attacks can cause many damages in recent planted seedlings. Control techniques and strategies should consider environmental, economical and social parameters, in the "Integrated Pest Management" context. The objective was to determine the viability utilization of the imidachlorprid molecule for protecting seedlings against pine aphid, using two different methods of application in the nursery of seedlings. The methodology consisted in a completely randomized design, with three treatments (imidachlorprid in the watering form; imidachlorprid associated to the soil conditioning gel; Control group) and six repetitions. The infestation was evaluated through the parameters incidence and prevalence. The residual effect of imidachlorprid application on seedlings in nursery (watering form and associated to the conditioning soil gel), is prolonged for 80 days after the seedlings planting. This effect does not impede the initial infestation, however is capable to avoid the population expansion of the colonies.

Key-words: Sistemic insecticide; imidacloprido; forest Integrated pest management.
\end{abstract}

\footnotetext{
${ }^{1}$ Engenheiro Florestal, MSc., Professor Colaborador do Departamento de Engenharia Florestal - Universidade Estadual do Centro Oeste do Paraná. PR 153 - Km 07 - Riozinho - Caixa Postal 21 - CEP 84.500-000 - Irati - Paraná. alvaro.faria@onda.com.br

${ }^{2}$ Engenheiro Florestal. Dr., Professor do Departamento de Ciências Florestais da UFPR. Centro de Ciências Florestais e da Madeira, Universidade

Federal do Paraná. Rua Lothário Meissner, 3400, 80210-170 - Jardim Botânico - Curitiba - Paraná. nsousa@floresta.ufpr.br
} 


\section{INTRODUÇÃO}

Segundo PENTEADO et al. (2000), Cinara pinivora (Wilson 1919), espécie nativa da América do Norte (leste dos Estados Unidos e Canadá), foi introduzida acidentalmente na Austrália, Argentina, Uruguai e Brasil, sendo o registro no Brasil feito por IEDE et al. (1998), no município de Lages - SC e Cambará do Sul - RS, atacando plantios de Pinus elliottii (Engelm) e Pinus taeda (L.), posteriormente se disseminando para os outros estados vizinhos. Em 1998, foi registrada a presença da espécie Cinara atlantica (Wilson, 1919) por LAZZARI e ZONTA-DECARVALHO (2000), e segundo PENTEADO et al. (2000) vem sendo encontrada em povoamentos de Pinus spp. nos estados do Rio Grande do Sul, Santa Catarina, Paraná, São Paulo até Minas Gerais.

Os pulgões do gênero Cinara (Curtis, 1835), atualmente destacam-se no cenário florestal brasileiro como uma importante praga para a cultura do pinus. Sua ocorrência é observada em plantas de todas as idades, porém danos mais severos incidem no momento do plantio, havendo indícios de que a associação entre pulgões, formigas e condições climáticas pode provocar a morte de mudas nos três primeiros meses após o plantio.

Para sua alimentação, os afídeos inserem o seu estilete na planta, até atingir o floema. A seiva elaborada é rica em açúcares e pobre em proteínas. Assim, estes insetos necessitam ingerir uma grande quantidade de seiva, para obterem a quantidade de aminoácidos necessária à sua sobrevivência. Desta forma, ingerem também uma grande quantidade de açúcares, que é eliminada na forma de "honeydew". Este, por sua vez, também pode ser utilizado como alimento por muitas espécies de insetos e fungos (ZALESKI, 2003). Para PENTEADO et al. (2000), as árvores atacadas por $C$. pinivora apresentam clorose, deformação e queda prematura das acículas, com redução no desenvolvimento da planta, entortamento do fuste e perda de dominância apical.

Os danos ficaram fortemente evidenciados em 2001, quando em algumas empresas florestais foram registradas mortalidades de mudas no campo, que geraram taxas de replantio da ordem de $17 \%$, representando prejuízos significativos. Em função deste quadro, a busca de alternativas para o controle do pulgão-do-pinus tornou-se uma necessidade e tem despertado o envolvimento de empresas e instituições de pesquisa, na busca por alternativas de "manejo integrado". Entre as alterativas disponíveis estão os inseticidas, porém, o sucesso no seu uso só será possível se a seleção dos princípios ativos for criteriosa e com a utilização de produtos seletivos (ou que sejam aplicados de forma direcionada), que não eliminem os inimigos naturais (predadores e parasitóides) do agrossistema.

Com base em testes de laboratório, FARIA e SOUSA (2005) concluíram que os inseticidas sistêmicos, especificamente os do grupo químico dos neonicotinóides, atenderiam a proposta do manejo integrado. Assim, a partir de aplicações em viveiro, seria possível obter em campo o efeito residual do produto, impedindo o ataque desta praga na fase de adaptação das mudas em campo, logo após o plantio. Desta forma, tornou-se necessária a realização de novos testes, em condições de campo, que confirmassem a persistência residual de oitenta dias do princípio ativo imidacloprido, aferida nos ensaios laboratoriais, e permitissem avaliar as melhores técnicas de aplicação do imidacloprido.

Com base na hipótese de que os tratamentos em viveiro podem conferir uma proteção de oitenta dias depois do transplantio, o objetivo deste trabalho foi determinar a viabilidade da molécula imidacloprido na proteção de mudas de Pinus taeda L. contra a presença do pulgão-do-pinus, sob duas modalidades de aplicação no viveiro de mudas.

\section{METODOLOGIA}

O experimento foi instalado em 18 de julho de 2002 no município de Três Barras, localizado na região do Planalto Norte catarinense (Estado de Santa Catarina), sob coordenadas geográficas de 2607'41" de latitude Sul e 5019'30" de longitude Oeste. O clima é temperado úmido CFb com invernos frios e chuvosos e precipitação média entre 1.200 a 1.600 $\mathrm{mm}$ anuais (Koeppen). O solo é do tipo Latossolo, com topografia plana.

Foi utilizado um delineamento experimental inteiramente casualisado com três tratamentos e seis repetições. Cada parcela de tratamento foi constituída de 36 mudas, dispostas em seis linhas paralelas de plantio, cada linha contendo seis plantas, num espaçamento de $2,5 \times 2,5 \mathrm{~m}$. Todas as mudas de Pinus taeda L. utilizadas foram de raiz nua, com oito meses de idade. Após o plantio verificou-se que as plantas possuíam 18,16 $\pm 1,33 \mathrm{~cm}$ de altura e 2,85 \pm $0,58 \mathrm{~mm}$ em diâmetro de colo, referente a toda população experimental (648 plantas).

Todas as mudas dos diversos tratamentos foram levadas do viveiro para o plantio sem a presença de pulgões, para que a infestação nas plantas ocorresse no campo.

\section{Tratamentos}

O tratamento 1 ("IMID") consistiu de plantas regadas com imidacloprido, ainda no viveiro, seis dias antes do plantio. Para a aplicação, utilizou-se um trator acoplado a um tanque de $400 \mathrm{~L}$ com uma barra adaptada à largura do canteiro. A velocidade do trator foi estabelecida de forma a irrigar $15 \mathrm{ml}$ de calda por muda. A concentração por litro foi de $0,315 \mathrm{~g}$ de ingrediente ativo (i.a.).

O tratamento 2 ("GEL+IMID") consistiu no acondicionamento do sistema radicial das mudas, em uma solução de gel condicionador de solo ${ }^{1}$ e imidacloprido diluído, imediatamente após a retirada das mudas nos canteiros. Assim, após a seleção e poda de raízes, as mudas foram tratadas com imersão da parte radicial em calda composta de gel 
condicionador de solo + imidacloprido por aproximadamente 60 segundos.

A concentração de gel utilizada foi de $1 \mathrm{~g}$ $\mathrm{L}^{-1}$, e a concentração de imidacloprido foi a mesma que a utilizada no "IMID" $\left(0,315 \mathrm{~g} \mathrm{~L}^{-1}\right.$ de i.a.). Para a aplicação foi adicionado em um balde com dois litros de água, duas gramas de gel e $0,9 \mathrm{~g}$ de produto comercial, sendo esta solução submetida à mistura até a plena homogeneização. Esta quantidade de calda foi suficiente para tratar todas as mudas desse tratamento.

Para o grupo controle (testemunha), as mudas utilizadas foram apenas submetidas à seleção e poda de raiz.

\section{Avaliações}

As avaliações ocorreram a cada 40 dias após o plantio, período este múltiplo de 80 dias, ou seja, o prazo de efeito residual do imidacloprido em mudas de Pinus taeda, estimado nos testes de FARIA e SOUSA (2005). Foram realizadas seis avaliações perfazendo um período de 200 dias.

A contagem de insetos em cada planta foi realizada de forma visual até o máximo de 20 insetos por planta. Este critério foi adotado em função da praticidade de execução da tarefa com os recursos financeiros, materiais e humanos disponíveis.

Utilizaram-se duas variáveis distintas, denominadas incidêndia e prevalência, para aferir o efeito do método de aplicação do inseticida e seu efeito residual no controle do pulgão-do-pinus.
Incidência foi estabelecida como número de plantas por parcela que continham entre um e vinte pulgões, indicando a taxa de infestação em cada avaliação.

Prevalência foi o número de plantas (casos) por parcela que continham mais de vinte pulgões, no momento da avaliação. Indicava a taxa na qual a infestação de pulgões teria condições de se estabelecer nas plantas e desenvolver suas colônias.

A utilização das variáveis incidência e prevalência permitu a avaliação dos tratamentos em função do número de plantas atacadas por parcela. Com estas variáveis, foi possível obter a homogeneização das variâncias entre as médias de cada tratamento, o que permitiu o uso da ANOVA e tornou possível a comparação destas médias pelo teste de Tukey a $5 \%$ de probabilidade.

\section{RESULTADOS E DISCUSSÃO}

\section{Incidência}

Na pré-avaliação (momento do plantio), não havia incidência de pulgões nas plantas. Após quarenta dias, entretanto, nenhum tratamento foi eficiente em impedir a ocorrência de pulgões-dopinus nas plantas $\left(F=1,58^{\text {ns }}, p<0,05\right)$. A incidência observada foi pequena, em termos absolutos. $O$ valor de $0,9 \%$ do "GEL+IMID" representa apenas duas mudas infestadas em 216 mudas, contra dez mudas do "IMID" e 12 mudas da testemunha (Tabela 1).

TABELA 1 - Incidência do pulgão-do-pinus em mudas de $P$. taeda em função do tratamento aplicado para seu controle e do período de permanência em campo. Três Barras, SC. 2002.

\begin{tabular}{|c|c|c|c|c|c|c|}
\hline Tratamento $^{\top}$ & Pré-avaliação & & 40 Dias & & 80 Dias & \\
\hline Testemunha & $0,0 \% \pm 0,00$ & - & $5,6 \% \pm 0,04$ & $\mathrm{a}$ & $6,5 \% \pm 0,02$ & a \\
\hline Tratamento 1 (“IMID”) & $0,0 \% \pm 0,00$ & - & $4,6 \% \pm 0,04$ & $a$ & $4,2 \% \pm 0,05$ & a \\
\hline Tratamento 2 ("GEL+IMID") & $0,0 \% \pm 0,00$ & - & $0,9 \% \pm 0,01$ & $\mathrm{a}$ & $0,9 \% \pm 0,01$ & a \\
\hline F (Trat.) & - & & \multicolumn{2}{|l|}{$1,58^{\mathrm{ns}}$} & \multicolumn{2}{|l|}{$2,48^{\text {ns }}$} \\
\hline Tratamento & \multicolumn{2}{|l|}{120 Dias } & \multicolumn{2}{|l|}{160 Dias } & \multicolumn{2}{|l|}{200 Dias } \\
\hline Testemunha & $19,0 \% \pm 0,02$ & $\mathrm{a}$ & $13,9 \% \pm 0,01$ & $\mathrm{a}$ & $17,1 \% \pm 0,01$ & a \\
\hline Tratamento 1 (“IMID”) & $19,9 \% \pm 0,07$ & a & $15,3 \% \pm 0,05$ & $\mathrm{a}$ & $20,4 \% \pm 0,09$ & a \\
\hline Tratamento 2 ("GEL+IMID") & $20,4 \% \pm 0,08$ & $a$ & $15,3 \% \pm 0,10$ & $\mathrm{a}$ & $21,3 \% \pm 0,07$ & a \\
\hline $\mathrm{F}$ (Trat.) & $0,04^{\mathrm{ns}}$ & & $0,04^{\mathrm{ns}}$ & & $0,39^{\mathrm{ns}}$ & \\
\hline
\end{tabular}

. As médias seguidas por letras diferentes na coluna diferem significativamente pelo Teste de Tukey a $5 \%$ robabilidade.

* = significativo; ns = não significativo). 
Na segunda avaliação, apesar de terem sido observados nos tratamentos inseticidas valores inferiores ao resultado da testemunha, constatou-se que o principio ativo imidacloprido, sob as duas formas de aplicação em teste, não impediu a colonização do pulgão-do-pinus nas plantas. Ou seja, aos oitenta dias, nas plantas do "IMID" constatou-se incidência em torno de 4\%, nas do "GEL+IMID", menos de $1 \%$, e nas mudas da testemunha, apesar do aumento da incidência de $5,6 \%$ para $6,5 \%$ $\left(F=2,48^{\text {ns }}, \quad p<0,05\right)$, não houve diferenças significativas. Estes resultados podem ser justificados por IEDE et al (1998) e PENTEADO et al. (2000), que mencionam que o pulgão-do-pinus se dissemina pelas correntes de vento. Ou seja, a populações de pulgões (Cinara spp.) podem alcançar as plantas, mesmo que em níveis insuficientes para prejudicarem seu desenvolvimento.
Nas demais avaliações, a incidência também não apresentou diferenças significativas entre tratamentos $\left(F=0,04^{\text {ns }}, 0,04^{\text {ns }} e 0,39^{\text {ns }}, p<0,05\right)$.

\section{Prevalência}

O tratamento com gel e imidacloprido ("GEL+IMID") foi comprovadamente eficiente em impedir que os pulgões incidentes nas plantas pudessem se desenvolver, pois após quarenta dias $\left(F=6,42^{*}, p<0,05\right)$ nenhuma planta do "GEL+IMID" apresentou colônias de pulgões com muitos individuos. Entretanto, existe forte indício de que o tratamento com rega de imidacloprido em viveiro ("IMID") impediu o desenvolvimento de colônias, porque o resultado de $0,5 \%$ das plantas representou apenas 1 em um total de 216 plantas avaliadas (Tabela 2).

TABELA 2 - Prevalência de colônias do pulgão-do-pinus em mudas de $P$. taeda em função do tratamento aplicado para seu controle e do período de permanência em campo. Três Barras, SC. 2002.

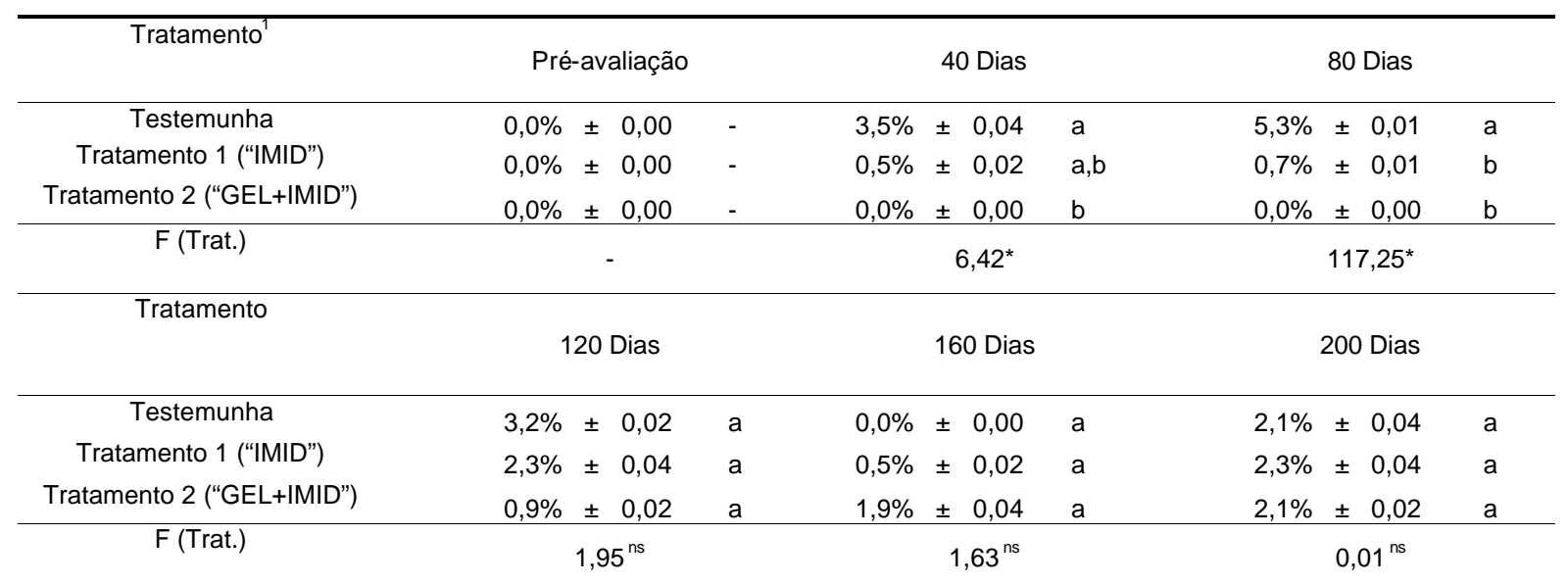

'. As médias seguidas por letras diferentes na coluna diferem significativamente pelo Teste de Tukey a $5 \%$ probabilidade.

( ${ }^{*}$ = significativo; $\mathrm{ns}=$ não significativo).

Na avaliação aos oitenta dias, as plantas do "GEL+IMID" impediram novamente o desenvolvimento das colônias $\left(F=117,25^{\star}, p<0,05\right)$, e as plantas do "IMID" manifestaram diferença significativa em relação à testemunha. Neste grupo, houve um aumento da prevalência de $3,5 \%$ para $5,3 \%$ das plantas (Tabela 2). Este aumento de prevalência coincidiu com as informações de IEDE (2003) e ESKIVISKI et al. (2003) e WILCKEN et al. (2003a), que constataram picos populacionais do pulgão-dopinus, no mês de outubro.

Os resultados referentes ao efeito residual do imidacloprido, detectados pela prevalência das colônias nas mudas, foram distintos dos resultados obtidos por WILCKEN et al. (2002) e WILCKEN et al. (2003b), que testaram os neonicotinóides imidacloprido e tiacloprido, além do organofosforado sistêmico acefato, obtendo efeito residual por 29 dias.
Alguns fatores podem estar relacionados a esta diferença, sendo um deles referente à metodologia adotada. Nos testes realizados por WILCKEN et al. (2002) e WILCKEN et al. (2003b) foram feitas aplicações diretamente no campo, inviabilizando comparações quanto às concentrações de ingrediente ativo por planta, em relação aos testes deste trabalho, em que o imidacloprido foi aplicado em viveiro e em duas formas distintas.

Outro fator a ser considerado é que a formulação comercial do imidacloprido utilizada no presente teste (grânulos dispersíveis em água), se diferenciou da formulação utilizada por WILCKEN et al. (2002), que utilizou este inseticida formulado como solução concentrada.

Outra diferença, diz respeito à forma de aplicação, que no caso dos trabalhos citados foi basicamente feita sob pulverização. Provavelmente este tipo de aplicação contribuiu para a diferença no 
efeito residual observada entre os testes, visto que em função da fotodegradação do imidacloprido (citado por WAMHOFF e SCHNEIDER, 1999), a melhor forma de aplicação em campo seria através de jato dirigido à base da planta.

Face à confrontação com resultados díspares, relatados na bibliografia, é de se supor que a formulação dos produtos a base de imidacloprido e as diversas metodologias de aplicação (rega, imersão, pulverização, pincelamento de tronco, entre outros) têm influência no efeito residual da molécula, e, portanto, comparações diretas de resultados referentes à eficiência e efeito residual devem ser feitas com cautela. Neste sentido, recomenda-se a realização de novos experimentos, no intuito de avaliar as melhores formulações e metodologias de aplicação deste princípio ativo em plantios recentes de pinus em campo.

Passados 120 dias, as plantas dos tratamentos inseticidas começaram a apresentar os mesmos níveis de prevalência do tratamento testemunha, ou seja, o nível de infestação das plantas não manifestou mais diferenças significativas entre os tratamentos até o final do experimento $\left(F=1,95^{\text {ns }}\right.$; $1,63^{\text {ns }} ; 0,01^{\text {ns }}, p<0,05$ para 120,160 e 200 dias, respectivamente). Desta forma, observou-se que o efeito da aplicação de imidacloprido no viveiro começou a ser minimizado, indicando que o efeito tóxico foi perdido em campo entre os oitenta e 120 dias após o plantio. Este resultado concorda com a conclusão de FARIA e SOUSA (2005) de que o efeito residual do imidacloprido alcança oitenta dias.

A utilização de defensivos sistêmicos e seletivos em viveiro - como é o caso do imidacloprido neste trabalho - para a proteção de mudas de pinus, apresenta diversas vantagens para o manejo integrado do pulgão-do-pinus, em relação a aplicações em campo. Primeiramente, restringe a área de aplicação à área do viveiro, representando em economia de tempo e mão de obra, menor risco de intoxicações e menor gasto com inseticidas. Também, os custos relacionados ao replantio são minimizados, como custo de mudas e mão de obra para o replantio. Em empresas que possuem viveiros com produção na ordem de milhões de mudas por ano, o custo com tratamentos discutidos neste trabalho, dificilmente ultrapassariam mais que um centavo de Real por muda. Além disso, a aplicação em viveiro favorece o recolhimento das embalagens do inseticida utilizado. Uma vez que ambas as formas de aplicação dos tratamentos inseticida utilizados neste ensaio foram viáveis, a escolha entre elas deverá ser fundamentada no modelo de produção de mudas, quer seja em raiz nua ou tubetes.

\section{CONCLUSÃO}

O efeito residual do imidacloprido aplicado sobre mudas em viveiro, quer na forma de rega ou associado ao gel condicionador de solo, se prolonga por até 80 dias após o plantio das mudas em campo. Este efeito não impede a infestação inicial das mudas pelo pulgão-do-pinus (Cinara spp.), porém é capaz de evitar a expansão populacional das colônias.

\section{AGRADECIMENTOS}

Os autores agradecem a colaboração no desenvolvimento deste trabalho à Fundação de Pesquisas Florestais do Paraná e à Rigesa S/A, por fornecerem suporte legal, financeiro e operacional para realização desta pesquisa.

\section{REFERÊNCIAS}

1. ESKIVISKI, E.; AGOSTINI, J., TOLOZA, R.; COOL, O. Biologia y efectos de Cinara spp. (Hemiptera: Aphididae) em plantaciones jovenes de Pinus em las províncias de misiones y corrientes, Argentina. In: SIMPÓSIO SOBRE CINARA EM PINUS, 1., 2003, Curitiba. Anais. Colombo: Embrapa Florestas, 2003. 1 CD-ROM.

2. FARIA, A.B.C.; SOUSA, N.J. Estratégias no manejo de resistência a inseticidas para o pulgão-do-pinus (Cinara pinivora Wilson e Cinara atlantica Wilson; Hemiptera: Aphididae). Floresta, v. 35, n. 1, p. 153-167, 2005.

3. IEDE, E.T.; LAZZARI, S.M.N.; PENTEADO, S.R.C.;ZONTA-DE-CARVALHO, R.C.; RODRIGUES-TRENTIN, R.F. Ocorrência de Cinara pinivora (Homoptera: Aphididae, Lachnidae) em reflorestamento de Pinus spp. no sul do Brasil. In: CONGRESSO BRASILEIRO DE ZOOLOGIA, 1998, Recife. Anais. Recife: Universidade Federal de Pernambuco, 1998. p. 141.

4. IEDE, E.T. Monitoramento das populações de Cinara spp., avaliação de danos e em plantios de Pinus spp. no sul do Brasil. Curitiba, 2003. 171 f. Tese (Doutorado em Ciências Biológicas) - Universidade Federal do Paraná.

5. LAZZARI, S.M.N.; ZONTA-DE-CARVALHO, R.C. Aphids (Homoptera: Aphididae) on Pinus spp and Cupressus spp in Southern Brazil. In: International Congress of Entomology, 21., 2000, Foz do Iguaçú. Anais. Foz do Iguaçu, 2000. 493 p.

6. PENTEADO, S.R.C.; TRENTINI, R.T.; IEDE, E.T.; REIS FILHO, W. Ocorrência, distribuição, danos e controle de pulgões do gênero Cinara em Pinus spp. no Brasil. Floresta, v. 30, n. 1/2, p. 55-64. 2000a.

7. PENTEADO, S.R.C.; QUEIROZ, E.C.; MESSA, R.C.; REIS FILHO, W.; IEDE, E.T. Biologia de Cinara atlantica (Homoptera: Aphididae: Lacchnae), em duas temperaturas, em laboratório. In: CONGRESSO BRASILEIRO DE ENTOMOLOGIA, 19; 2002, Manaus. Livro de Resumos. Manaus: Sociedade Entomológica do Brasil: 2002. p. 34.

8. WAMHOFF, H.; SCHNEIDER, V. Photodegradation of imidacloprid. Journal of Agricultural and Food Chemistry. v. 47, n. 4,p. 1730-1734.1999.

9. WILCKEN, C.F.; OLIVEIRA, N.C.; OTATI, A.L.T.; COUTO, E.B.; SANTOS, T.G.; SOUZA, A.P. Controle do pulgão dos pinheiros Cinara atlantica (Hemíptera: Aphididae) em plantios de Pinus, com inseticidas cloronicotinis. In: CONGRESSO BRASILEIRO DE ENTOMOLOGIA, 19; 2002, Manaus. Livro de Resumos. Manaus: Sociedade Entomológica do Brasil, 2002. p. 153. 
FARIA, A.B.C. e SOUSA, N.J. Efeito residual em campo...

10. WILCKEN, C.F.; OLIVEIRA, N.C.; OTATI, A.L.T.; COUTO, E.B.; FERREIRA FILHO, P.J. Dinâmica da infestação de Cinara atlantica em diferentes espécies de Pinus em Buri, SP. In: SIMPÓSIO SOBRE CINARA EM PINUS, 1. 2003, Curitiba. Anais. Colombo: Embrapa Florestas, 2003a. 1 CD-ROM.

11. WILCKEN, C. F.; OLIVEIRA, N.C.; OTATI, A.L.T. Ações de pesquisa visando o manejo integrado dos pulgões-gigantes-dopinus em São Paulo. In: SIMPÓSIO SOBRE CINARA EM PINUS, 1. 2003, Curitiba. Anais. Colombo: Embrapa Florestas, 2003b. 1 CD-ROM.

12. ZALESKI, S.R.M. Biologia, danos e determinação dos limites térmicos para o desenvolviemento de Cinara atlântica (Wilson, 1919) (Hemiptera: Aphididae) em Pinus taeda L. (Pinaceae). Curitiba, 2003. Dissertação (Mestrado em Entomologia). Universidade Federal do Paraná. Curitiba, 2003.

Recebido em 21/12/2005

Aceito em 05/07/2007 